BULLETIN Bulletin hispanique

HISPANIQUE Université Michel de Montaigne Bordeaux

112-1 | 2010

Actes du Colloque « langue, littérature, littéralité »

\title{
Autoridad y poder en el Siglo de Oro
}

Universidad de Navarra, Iberoamericana-Vervuert, Madrid, Frankfurt am Main, 2009

Jean-Pierre Dedieu

\section{(2) OpenEdition}

1 Journals

Édition électronique

URL : http://journals.openedition.org/bulletinhispanique/1196

DOI : 10.4000/bulletinhispanique.1196

ISSN : 1775-3821

Éditeur

Presses universitaires de Bordeaux

Édition imprimée

Date de publication : 1 juin 2010

Pagination : 443-445

ISBN : 978-2-86781-692-5

ISSN : 0007-4640

Référence électronique

Jean-Pierre Dedieu, «Autoridad y poder en el Siglo de Oro », Bulletin hispanique [En ligne], 112-1 | 2010, mis en ligne le 04 janvier 2013, consulté le 23 septembre 2020. URL : http://journals.openedition.org/ bulletinhispanique/1196 ; DOI : https://doi.org/10.4000/bulletinhispanique.1196 
pas. Quel beau sujet eût fait l'étude de la construction d'un tel mythe de décadence ! Ce n'était pas la question posée. L'efficacité pédagogique commandait de la taire.

Jean-Pierre DEDIEU

Autoridad y poder en el Siglo de Oro. Ignacio Arellano, Christoph Strosetzki, Edwin Williamson (eds.). - Madrid, Frankfurt am Main, Universidad de Navarra / Iberoamericana / Vervuert, 2009, 293 p. (Biblioteca Áurea Hispánica, 62) : ISBN 978-84-8489-470-4.

Il s'agit de la publication de quinze communications faites aux séminaires d'un groupe de discussion organisé par des chercheurs des Universités de Navarre, Münster et Oxford, autour des rapports entre pouvoir politique, autorité et vie littéraire au XVII siècle.

Les textes traitent pour moitié d'une problématique directement littéraire: le Quichotte (Edwin Williamson, María del Carmen Rivero Iglesias), Garcilaso (Colin Thompson), le Siglo pitagórico de Enríquez Gómez (Cerstin Bauer Funke), le théâtre de Juan de la Encina (Tobias Leuker), le roman picaresque (Michaela Peters), Calderón (Victoriano Roncero) et Lope de Vega (Jonathan Thacker), le genre de l'emblème (Rafael Zafra). D'autres sont directement historiques : la figure du roi dans les fêtes hagiographiques françaises (Ignacio Arellano), les rapports de la littérature avec la politique (Luis Galván), le concept de loi et de droit (Christoph Strosetzki), le travail de contrôle des librairies par l'inquisition (Ronald Truman), le cérémonial diplomatique (Thomas Weller), l'image de la mort du roi (Ulrich Winter).

Il m'est difficile d'évaluer la pertinence de ces analyses sous l'angle strictement littéraire. Toutefois l'ambition des auteurs porte non pas sur celui-ci à proprement parler, mais sur les rapports de la production littéraire avec le politique. C'est donc cet aspect qui me retiendra.

Premier constat, ces textes ont totalement assimilé un point capital de la théorie du pouvoir dans l'Espagne du XVII siècle qui échappe encore à beaucoup d'historiens : la limitation du pouvoir royal par le droit naturel et le droit divin, et la pénétration de cette idée dans la société, qui en fait une convention de base du politique, partagée par l'ensemble de la population. Le texte de Strosetzki, par exemple, en traite directement, ceux de Peters, de Carmen Rivero et de Roncero en présupposent la diffusion. Le travail fascinant de Williamson en fait même l'articulation principale du Quichotte, dans lequel il voit une parabole politique fondée sur l'évolution des rapports entre le héros éponyme et Sancho. On regrettera cependant 
qu'une fois décrits les principes théoriques de cette conception du politique, les auteurs s'intéressent si peu à leur mise en œuvre pratique, notamment au rôle de l'Église comme gardienne des règles. Rien sur les universités, sur la production directe de théories politiques, sur la prédication, sur le rôle des intellectuels - à cette époque, des clercs - comme experts politiques. Ce n'est pas leur sujet, dira-t-on. Voire. Il n'en reste pas moins qu'il y a des questions qu'il est difficile de traiter en s'en tenant au canon littéraire des auteurs consacrés.

Deuxième constat, beaucoup de ces textes tentent une approche réelle du thème de l'ouvrage. Le plus ambitieux est certainement celui de Luis Galván, qui montre bien la complexité des rapports entre pouvoir politique et vie littéraire et comment la critique littéraire au pouvoir constitue un antécédent de l'opinion publique. Pour ce qui est des rapports du créateur à l'autorité intellectuelle, l'autre aspect de la question abordé dans ces textes, on trouvera des remarques intéressantes chez Bauer Funke, Thompson ou Zafra, pour ne citer qu'eux. Le problème, cependant, n'est nulle part abordé directement dans toute son ampleur.

Les travaux proprement historiques sont de bonne facture. Ainsi se réjouira-t-on de l'effort de comparatisme qui conduit à traiter aussi de la France dans l'un des travaux présentés. Tout en saluant l'observation très juste du rôle capital du roi dans la mise en scène de la propagande catholique, phénomène que seul sans doute un étranger à notre nation pouvait faire avec une telle acuité, on regrettera le manque de mise en contexte par rapport aux débats qui agitaient alors l'Église de France. Y procéder aurait, sur ce point au moins, permis à l'auteur d'enrichir sa problématique. L'articulation entre les contributions d'histoire des représentations politiques et des études littéraires est réussie, du fait de la cohérence de la problématique abordée et de la manière de l'aborder. Certainement pas du fait du travail des éditeurs scientifiques.

Celui-ci est réduit à sa plus simple expression : une grosse page d'introduction plus institutionnelle qu'autre chose, et le classement des textes par ordre alphabétique du nom de famille des auteurs. Il est difficile de faire moins. Aucune définition de la problématique, aucune mise en contexte historiographique, aucune conclusion. Le texte brut. S'y reconnaisse qui pourra.

C'est dommage, car encore une fois le sujet est très intéressant et l'apport de chaque auteur, nécessairement partiel, réel. Le livre aurait vu sa valeur multipliée par une solide introduction (ou conclusion) thématique qui aurait systématisé les observations présentées. Presque tous ces textes, y compris ceux auxquels nous n'avons pas fait un sort particulier, méritent lecture ne serait- 
ce que pour leur caractère stimulant, tant pour les spécialistes de littérature que pour les historiens, qui pourront mesurer à travers le prisme littéraire l'importance de phénomènes dont ils ne sont pas toujours conscients.

Jean-Pierre DEDIEU

Fernanda Macchi, Incas ilustrados. Reconstrucciones imperiales en la segunda mitad del siglo XVIII, Madrid, Frankfurt, Vervuert-Iberoamericana, 2009, 286 p.

Incas ilustrados. Reconstrucciones imperiales en la segunda mitad del siglo XVIII est un ouvrage multiple comme l'indique le titre choisi par Fernanda Macchi. Elle analyse d'abord les rééditions et les traductions de l'œuvre de l'inca Garcilaso de la Vega, puis des écrits parus au XVIII' siècle évoquant l'ancien empire. D'origine argentine, Macchi est professeur à l'Université Mac Gill (Montréal) ; le corpus sur lequel porte son analyse est plurilingue (espagnol, français et anglais).

Garcilaso de la Vega, fils d'un conquérant et d'une princesse inca, partit en Espagne en 1560 pour revendiquer son héritage ; il vécut en Andalousie jusqu'à sa mort en 1616. La première partie de ses Commentaires royaux, recréant l'univers inca soixante ans après l'arrivée de Pizarre (1532), parut à Lisbonne en 1609, tandis que l'Histoire générale du Pérou, la deuxième partie des Commentaires consacrée à l'étape de la conquête et aux guerres intestines, fut éditée en 1617.

Fernanda Macchi relève un difficile pari : démontrer comment l'œuvre centrale de l'inca a été altérée au fil des éditions, soumise à une infinité de manipulations idéologiques, indécelables par le lecteur non spécialiste. Elle observe que le renouvellement des études coloniales, notamment grâce à la rigueur de chercheurs latino-américains (Mazzotti, García Bedoya, ChangRodriguez...), est méconnu au-delà des universités américaines, tandis que des travaux conçus sur la base de traductions anglaises et françaises ignorant l'histoire textuelle des Commentaires ont bénéficié d'une diffusion excessive. C'est contre cette manipulation des sources que s'insurge l'auteur d'Incas ilustrados en signalant: " ese tipo de lectura reproduce inconscientemente una lógica colonial » (86).

Après l'édition princeps, il fallut attendre plus d'un siècle, jusqu'en 1722 pour que les œuvres de Garcilaso paraissent à Madrid, sous l'autorité d'Andrés González de Barcia, dont Macchi s'attache à rappeler l'itinéraire dans la république des lettres de la première moitié du XVIII siècle. Barcia exerça de nombreuses missions au service de Philippe $V$ et prit part à la 